\title{
Europa - Ein Kontinent auf der Suche nach seiner Identität
}

Ein politisches Beben hat den Kontinent erfasst. Als seismographische Hilfe mag ein Blick auf die täglichen Schlagzeilen zur Krise genügen: „,Der Riese taumelt in sein Schicksal“, „Der Albtraum“, „Das doppelte Chaos“, „Im brennenden Haus“, „Rettung der Europäischen Idee“, „Europa ist müde“, „Katastrophenstimmung in Europa“. Solche Überschriften beschreiben die Bandbreite des aktuellen Befundes. $\mathrm{Da}$ ist ein großes politisches System orientierungslos geworden. Seine Prozeduren können die Krisen nicht meistern. Das Defizit besteht nicht nur in fehlender Bewältigung alltäglicher Detailprobleme. Vielmehr stellt sich die Frage nach Ziel und Zweck, ja nach der Sinnhaftigkeit des Projekts „Europa“. Der Philosoph Jürgen Habermas erklärt und deutet die Krise der Europäischen Union wie folgt: „Europa verharrt in einer Schreckstarre".

In der Geschichte der Europäischen Integration ist es nicht das erste Mal, dass in einer Krise die Frage nach der Sinnhaftigkeit gestellt wird. Bisher gab es jedoch immer eine Antwort, die dem Projekt immense Vitalität verlieh. Dies bleibt aber heute aus. Das kennzeichnet die neue Epoche - die Abwesenheit einer identitätsstiftenden Zielprojektion. Ohne Identitätsgrundlage fehlt aber jedem politischen System die Basis seiner Handlungsfähigkeit. Halten wir uns die bisherigen Phasen vor Augen:

- Unmittelbar nach dem Zweiten Weltkrieg war der Kontinent von einem Netz europäischer Bürgergruppen durchwebt, die eine große Lehre aus der Geschichte ziehen wollten. Kriege und Katastrophen nationalistischer Art sollten nicht mehr das letzte Wort persönlicher Erfahrung sein. Jetzt sollte dieser von breiten Blutspuren gekennzeichnete Kontinent die Alternative zum Nationalismus anstreben und verwirklichen: die Einigung Europas.

Man gab sich höchst anspruchsvolle Ziele. Dazu gehörte der europäische Bundesstaat, die Politische Union. Hatten doch bereits viele Widerstandsgruppen im Dritten Reich darüber nachgedacht und konzeptionelle Entwürfe geliefert. Aber diese große Ambition endete zunächst in einem bescheidenen Schritt. Nicht zuletzt hatte Großbritannien dafür gesorgt. Im Jahr 1949 wurde schließlich der Europarat gegründet. Das aber war nicht der große supranationale Wurf. 
- Man bediente sich einer anderen Methode um das Ziel zu erreichen. Es sollten bescheidene einzelne Funktionen integriert werden. So legten Robert Schuman und Jean Monnet funktionalistische Konzepte für eine Europäische Gemeinschaft für Kohle und Stahl vor. So konnte auch der frühere Kriegsgegner Deutschland weiter kontrolliert werden ohne diskriminiert zu sein.

- Als dies so eindrucksvoll gelang, galt es sogleich wichtige Funktionen nachzuschieben: die Organisation der Sicherheit (Europäische Verteidigungsgemeinschaft, EVG). Über solche funktionalistische Ansätze sollte aber ein großes politisches Dach gebaut werden - die Europäische Politische Gemeinschaft (EPG). In jeder Phase hatte man ein großes, präzises Ziel vor Augen.

- Das half auch über die Krise des Scheiterns von EVG und EPG hinweg. Funktionalistische Ersatzlösungen wurden in Auftrag gegeben. Nicht nur, dass die mit Souveränität auszustattende Bundesrepublik Deutschland sicherheitspolitisch dann eben anders verankert wurde, nämlich innerhalb von NATO und WEU. Man verhandelte - im sogenannten „Geist von Messina“ - um die Europäische Wirtschaftsgemeinschaft (EWG) und die Europäische Atomgemeinschaft (EURATOM). In der klaren Zielperspektive wurden auch die elementaren Konflikte zwischen Frankreich und der Bundesrepublik Deutschland lösbar. Frankreich gestaltete einen Gemeinsamen Markt, so wie es Bonn wollte, und die Bundesrepublik Deutschland gestattete das Herausnehmen der militärischen Komponente aus der nuklearen Kontrolle, so wie es Paris wollte. Der höchst ambitionierte Zielkorridor der Römischen Verträge sollte der Verwirklichung zugeführt werden.

- Ein erster größerer Zieldissens führte folgerichtig zur ersten Integrationskrise. Die USA und Großbritannien signalisierten ihre Bereitschaft, dem Druck der Sowjetunion auf den Status Berlins nachzugeben. Das bedeutete für Adenauer und de Gaulle eine Existenzbedrohung des freiheitlichen Westeuropas. Eine Politische Union mit sicherheitspolitischer Komponente sollte eine Antwort bieten. So wurde es in den sogenannten Fouchetplänen niedergelegt. Die übrigen EWGStaaten aber wollten dieser Führungsvorgabe - inzwischen misstrauisch geworden - nicht folgen. Adenauer und de Gaulle kreierten eine kleine Ersatzlösung, die aber sogar in der deutschen Innenpolitik auf Unverständnis stieß: der deutschfranzösische Freundschaftsvertrag.

- Die nachlassende Zielsicherheit für das Projekt „Europa“ führte zu etlichen Jahren der Stagnation und Krise. Die „Politik des leeren Stuhls“, die mit dem sogenannten „Luxemburger Kompromiss“ gewissermaßen beigelegt wurde - auch wenn der Interpretationsdissens blieb -, bot die symbolische Botschaft zur Lage. 
Diese unklare, verwirrende Perspektive ließ trotz des weiter bestehenden OstWest-Konflikts keine Integrationsdynamik im alten Stil mehr zu.

- Die krisenhafte Zuspitzung des Niedergangs fand eine scharfe und weithin akzeptierte Bezeichnung: „Eurosklerose“. Ende der 1970er, Anfang der 1980er Jahre befand sich die Europäische Integration in der Ära eines tiefen Niedergangs. „Eurosklerose“ wurde zum Schlüsselbegriff der Lagebeschreibung. Europa konnte mit den dynamischen Märkten nicht mehr mithalten. Es erschien erschöpft, gleichsam ein Ausschnitt aus dem Museum. Bundeskanzler Helmut Kohl und Staatspräsident François Mitterand erkannten die dringende Notwendigkeit eines strategischen Aufbruchs. Dazu bedurfte es eines entsprechend begabten politischen Kopfes. Sie fanden ihn in Jacques Delors. Er war ein starker französischer Finanzminister und die meisten sahen in ihm den zukünftigen französischen Staatspräsidenten. Er aber nahm die Herausforderung Europa an. Zunächst teilte er den Staats- und Regierungschefs mit, er müsse nun strategisch nachdenken. Nach einigen Monaten trug er sein Ergebnis vor: Europa braucht zum Aufbruch eine große historische Aufgabe. Dies könnte die Neuorganisation der Sicherheit oder die Vollendung des Binnenmarktes sein. Nur für eine dieser großen Aufgaben besitze Europa die Kraft. Der Binnenmarkt wurde als strategisches Thema angenommen. Dies bedeutete die mehrjährige Umsetzung von fast 300 Gesetzeswerken. Die Öffentlichkeit wurde überzeugt durch die Daten und Argumente des umfangreichen Cecchini-Reports. Der eingeschlagene Kurs wurde politisch über etliche Jahre durchgehalten.

Aus diesem gelungenen Beispiel ist für die gegenwärtigen Herausforderungen zu lernen: Europa braucht starke politische Führungsfiguren und strategische Köpfe. Die Politik muss die notwendigen Schritte strategisch erklären und vertrauensbildend durchhalten. Die Schlussfolgerung liegt auf der Hand: Europas Politik muss das Erklärungsdefizit eliminieren. Es ist viel mehr Zeit und Kraft auf die Erläuterung zu richten. Wer die Deutungshoheit gewinnt, der gewinnt auch die Zukunft.

Und dennoch: Auch ein strategischer Entwurf zum Projekt Europa wird heute in einen schwierigen Kontext geraten. Die sowieso nur schwach ausgeprägte Identität Europas hat inzwischen ihre politische Stabilisierungsleistung weitergehend eingebüßt. Es gab in früheren Jahrzehnten durchaus stärkere Prägungen.

Nach dem Zweiten Weltkrieg spürten die Europäer - jenseits ihrer entfernten historischen Erfahrungslinien - die ausgeprägte Notwendigkeit, als Antwort auf die Weltkriege eine Friedensgemeinschaft aufzubauen. Diese gemeinsame Idee geriet dann in einen dramatischen weltpolitischen Konflikt. Die Auseinandersetzung zwischen Ost und West war mehr als ein bloß vordergründiger Machtkonflikt: Sie war 
auch ein elementarer Konflikt um Normen, vornehmlich der Antagonismus von Freiheit und Unfreiheit. Sie war aber auch ein Konflikt zwischen Menschenbildern, vornehmlich der Antagonismus des Menschen als Person und des Menschen als Gattungswesen. Alles, was in Europa in diesen Jahrzehnten passierte, konnte in diese Antagonismen eingeordnet und so sofort erklärt werden. Als diese identitätsstiftenden Stützen mit Mauerfall und Untergang der Sowjetunion wegfielen, halfen zunächst die starken Erinnerungen. Außerdem wurden gewisse Brücken in die neue Zeit geschlagen: Das reichte vom Binnenmarkt bis zur Wirtschafts- und Währungsunion, von der EU-Osterweiterung bis zu markanten Vertragsreformversuchen. Aber inzwischen ist dies alles konsumiert. Die europäischen Erfolgsgeschichten sind zu Selbstverständlichkeiten degeneriert und daher im Blick auf Identitätshorizonte irrelevant. Es bleiben im Alltag viele Beschwerden und Frustrationen über bürokratische Monstererfahrungen Brüsseler Konvenienz. Europa erscheint in den täglichen Schlagzeilen zu den vielfältigen Krisenphänomenen. Nun versuchen viele Kräfte in Europa, mit populistischen Anti-Europa-Sprüchen politische Pluspunkte und Wahlerfolge zu sammeln. Aus diesem Defizit an europäischer Identität entstehen die ernsten Schwierigkeiten einer neuen Ära: Jedes politische System bedarf zu seiner Handlungsfähigkeit eines Orientierungsrahmens, auf den sich die Erklärungen und Interpretationen sowie die Begründungen für Prioritäten und Positionen beziehen.

In keinem politischen System existiert eine politische Ratio gleichsam als Ding an sich, ohne Bezugnahme auf einen elementaren Konsens, auf gemeinsame Erfahrungen und Interessen. Man mag es politische Kultur nennen, mag es kollektives Selbstverständnis, man mag es Identität nennen. Europa kann auf diese Ressource gemeinsamer Selbstwahrnehmung aber nur sehr begrenzt zurückgreifen. Natürlich existieren auch hier gemeinsame Erfahrungen, die Ablagerungen einer konfliktreichen Geschichte und die Erlebnisse gemeinsamer Erfolge. Aber diese Schicht europaweiter Gemeinsamkeiten bleibt vergleichsweise dünn. Sie reicht um einen gemeinsamen Markt zu begründen. Aber sie offenbart ihre Schwäche bei jedem Schritt, der darüber hinausgeht.

Die Europäer erzählen sich nicht eine gemeinsame Geschichte, sie verfügen nicht über ein Narrativ. Selbst die traumatische Erfahrung der Rückkehr des Krieges auf den Balkan in den 1990er Jahren wurde nicht gemeinsam verarbeitet, sondern in getrennten nationalen Erlebniskulturen - in Großbritannien anders als in Deutschland, in Frankreich anders als in Italien. Das gilt auch für andere große Themen von der Wirtschafts- und Währungsunion bis zur Verfassungsfrage.

Ohne einen solchen Kontext der europäischen Selbstverständigung fehlen für den europapolitischen Kurs der Kompass und das stützende Geländer. Alles wird zum 
situationsorientierten Basarhandel - wie wir es von den Gipfelkonferenzen kennen. Dies ist jedoch nicht wie eine naturgesetzliche Zwangsläufigkeit über uns gekommen, sondern auch der Reflex einer jahrzehntelangen Vernachlässigung europäischer Orientierungsdebatten. Ein Walter Hallstein konnte noch vom ,unvollendeten Bundesstaat“, ein Leo Tindemans von der „vorhandenen europäischen Identität“, ein Joschka Fischer von der „Finalität Europas“" sprechen. Dies alles erscheint uns heute wie ein Echo aus einer weit entfernten Epoche.

Der aktuelle Befund lautet daher: Europa braucht Ziele, Perspektiven, Orientierungen. Es muss eine strategische Kultur aufbauen.

Grundlage für einen solchen politisch-kulturellen Schritt bietet eine deutlich gesteigerte Handlungsfähigkeit der Europäischen Union - wozu die Führungsarchitektur einer transparenten Klärung bedarf. Bei den Verhandlungen um den Vertrag von Lissabon hatte man das Problem erkannt, Korrekturversuche unternommen, aber keine wirkliche Lösung herbeigeführt. Seit Inkrafttreten des Lissabon-Vertrags 2009 gibt es ein politisches Konsortium, das sich die Führung teilt: der auf maximal zwei mal 2,5 Jahre gewählte Präsident des Europäischen Rates, der auf fünf Jahre gewählte Präsident der Kommission, der für sechs Monate im Amt befindliche Präsident des Ministerrates, der Vorsitzende der Euro-Gruppe, der Hohe Repräsentant für Außen- und Sicherheitspolitik. Ungeklärt bleibt jedoch bis heute, wer aus diesem Quintett welchen Führungsauftrag gegenüber wem wahrzunehmen hat. In diesen Dschungel von Führungsverantwortungen mischen sich die Staats- und Regierungschefs der großen Mitgliedsstaaten und inzwischen auch das selbstbewusster auftretende Europäische Parlament ein. Ein effektiver, zielführender Entscheidungsprozess ist auf diese Weise nicht zu organisieren. Der Umgang mit der Finanzkrise liefert dazu empirisches Datenmaterial in Hülle und Fülle.

Es wäre nun naiv, einfach auszurufen „Europa braucht einen neuen Vertrag“. Nach den vielfältigen Verhandlungen seit dem Vertrag von Nizza sind dazu die Kräfte erschöpft. Es würde auch - ohne Vertragsänderung - ausreichen, eine machtpolitische Verständigung darüber herbeizuführen, wer denn nun in der EU das Sagen hat. Wenn dies nicht gelingt, dann wird Europa sich mehr und mehr in nationale Egoismen zurückziehen und seine Substanz zerbröseln. Es kommt nicht von ungefähr, dass die klassischen, traditionellen Europapolitiker inzwischen Sorge um Sorge mit Blick auf Europa äußern. Sie sehen den großartigen ideellen Horizont ihres „alten Europa“" gefährdet.

Bei allen Krisenmeldungen darf man aber auch die Lernprozesse nicht übersehen, die unter Druck und im Stress ausgelöst werden. Die Wirtschafts- und Währungsunion ist hierfür das beste Beispiel. Im Vertrag von Maastricht hatte man zwar die Währungsunion präzise mit ihren Stabilitätskriterien definiert. Die von Anfang an 
geforderte politische Umrahmung hatte man jedoch ausgespart. Auch wurde erst später bemerkt, dass die Stabilitätskriterien nur bis zur Einführung der gemeinsamen Währung gelten sollten. Aber was sollte anschließend geschehen? Flugs schob man den Stabilitätspakt nach, der die Kriterien für die Zeit danach fixierte. Eine EuroGruppe hatte der Maastricht-Vertrag auch nicht vorgesehen. Man schob sie informell nach. Im Lissabon-Vertrag wurde die Euro-Gruppe dann vertraglich formalisiert. Die Elemente einer Politischen Union, einer Wirtschaftsregierung, wurden unter dem Druck der Finanzkrise wieder thematisiert. Die Kontrollkompetenzen der Europäischen Union wurden ebenso ausgebaut wie die europäischen Instrumente zur Währungsstabilisierung. Der Krisendruck erweist sich somit immer wieder als ein zentrales Instrument zur Fortentwicklung der Integration.

Auf der Grundlage von Druckkonstellationen wird Europa eine weitere strategische Perspektive ausbauen: die „differenzierte Integration“. Diese Herausforderung kombiniert Fragen der Führungsstrategie mit Fragen der Identität. Eine Vertiefung der Integration im Gleichschritt wird zwar immer schwieriger zu bewerkstelligen sein. Entscheidend ist es jedoch, diese Tatsache nicht allein als Problem, sondern auch als strategische Chance für die Zukunft Europas zu sehen.

Bereits in den neunziger Jahren des letzten Jahrhunderts, als mit der Wirtschaftsund Währungsunion und dem bevorstehenden Beitritt neuer Mitgliedstaaten aus Mittel- und Osteuropa Strategien zur Vertiefung und Erweiterung parallel verfolgt wurden, besannen sich Europas Spitzenpolitiker einer Idee, die Willy Brandt und Leo Tindemans bereits zwanzig Jahre zuvor geprägt hatten: die differenzierte Integration. In den verschiedenen Politikbereichen sind seitdem Integrationsschritte erfolgt, an denen sich nicht alle EU-Mitgliedsstaaten beteiligen. Die Eurozone, der Schengen-Raum und weitere Projekte zeigen, dass die differenzierte Integration schon seit etlichen Jahren ein fester Bestandteil des Integrationsprozesses ist.

Primärrechtlich wurde die differenzierte Integration erstmalig im Vertrag von Amsterdam verankert. Die Regeln dafür waren kompliziert und in der Realität nicht anwendbar. Dies hat man erkannt und die Regeln und Anwendungsbereiche von Nizza über den gescheiterten Verfassungsvertrag bis zum Vertrag von Lissabon verändert und es damit eher ermöglicht, Schritte der differenzierten Integration zu gehen. Man hat diese Möglichkeit im neuen Vertrag mit der „Ständigen Strukturierten Zusammenarbeit" sogar auf die Verteidigungspolitik ausgeweitet. Trotz des nach wie vor schwierigen Procedere war die Anwendung der differenzierten Integration nie einfacher als heute.

Differenzierte Integration kann als Laboratorium für das Innovationspotenzial der EU dienen. Die Heterogenität und die schiere Zahl unterschiedlicher Interessen laden geradezu dazu ein, Projekte voranzutreiben, die von einer Gruppe von Staaten 
für wichtig erachtet werden, die aber keine Realisierungschance im Geleitzug der ganzen Union haben. Im Umfeld der differenzierten Integration kursiert eine Vielzahl von Schlagworten und Leitbildern, von der abgestuften Integration über ein „Europa à la carte“ bis hin zum Gedanken eines Kerneuropas. Ein zukunftsfähiges Modell der Differenzierung muss sich an der Vorstellung eines offenen Gravitationsraumes orientieren. Sowohl ein fester und geschlossener Kern von Mitgliedsstaaten, der stets gemeinsam voranschreitet, als auch die Beliebigkeit unbegrenzter Wahlmöglichkeiten würden zwangsläufig eine Spaltung der Union herbeiführen.

Differenzierte Integration bedeutet eben gerade nicht, eine Zweiklassengesellschaft der europäischen Staaten einzuführen. Stattdessen sollten dort, wo eine Vertiefung gegenwärtig nicht mit allen Mitgliedsstaaten erfolgen kann, gezielt sachorientierte Kooperationsformen entstehen. Ist ein solches Projekt dann erst einmal erfolgreich umgesetzt, wird dieses die notwendige Anziehungskraft für den Beitritt weiterer Staaten entwickeln. Differenzierung in diesem Sinne ist also vor allem zeitlich beschränkt zu sehen. Das heißt keine dauerhafte Trennung konkurrierender Integrationsräume, sondern verschiedene Differenzierungsinitiativen, die sich nach und nach auf die ganze Europäische Union überführen lassen. Differenzierte Integration ist also keine Gefahr, sondern eine Chance.

Die Konsequenz aus alledem zu Zustand, Zukunft und Identität Europas lautet: Wer europäische Handlungsfähigkeit optimieren will, der muss nicht nur von institutionellen Reformen sprechen, sondern sich auch den Mühen europäischer Selbstverständigung unterziehen. Die politischen und kulturellen Eliten müssen ihr Verständnis der Risiken und Chancen ineinander verweben. Es geht also bei näherem Hinsehen nicht nur um Potentiale und Institutionen, sondern um die Grundlagen der politischen Kultur. Auch diese Dimension kann und muss man pflegen und organisieren. Die Mühe der Vorverständigung und der strategischen Zukunftsperspektive muss man in Europa auf sich nehmen, will man nicht immer wieder infantil beginnen und die alten Fehler wiederholen.

Korrespondenzanschrift:

Prof. Dr. Dr. h.c. Werner Weidenfeld

Centrum für angewandte Politikforschung $(C \cdot A \cdot P)$

Geschwister-Scholl-Institut für Politikwissenschaft

Ludwig-Maximilians-Universität München

Maria-Theresia-Str. 21

81675 München

E-Mail: werner.weidenfeld@1rz.uni-muenchen.de 